\title{
Dronabinol-induced hypomania: A case report and literature review
}

\author{
Shirshendu Sinha ${ }^{1}$, Audrey Umbreit ${ }^{2 *}$ and Charles Sieberg ${ }^{3}$ \\ ${ }^{1}$ Department of Psychiatry and Psychology, Mayo Clinic Health System, Southwest Minnesota region, Mankato, MN, USA \\ ${ }^{2}$ Department of Pharmacy, Mayo Clinic Health System, Southwest Minnesota region, Mankato, MN, USA \\ ${ }^{3}$ College of Pharmacy Postgraduate Residency Program, University of Minnesota, New Ulm, MN, USA
}

\section{Purpose}

Dronabinol is a synthetic derivative of cannabis that is commonly prescribed for chemotherapy-induced nausea and vomiting or cachexia due to HIV/AIDS. Dronabinol and the cannabinoids found in medical marijuana have complex effects on the central nervous system that can lead to both positive and negative patient outcomes. Here we present a case of dronabinol-induced hypomania in a 55-year-old male with a history of bipolar disorder and substance use disorder. This case emphasizes the need to thoroughly evaluate mental health conditions before prescribing medical marijuana or synthetic cannabis derivatives such as dronabinol.

\section{Background}

Medical cannabis is used for a variety of conditions including chemotherapy induced nausea and vomiting, appetite stimulation in HIV/AIDs, chronic pain, and spasticity [1]. The safety of medical cannabis as well as FDA-approved cannabinoids, dronabinol and nabilone, in those with bipolar disorder warrants further investigation as previous studies suggest that the use of cannabis may be associated with exacerbation of manic symptoms [2]. Medical cannabis is available in several formulations that contain varying amounts of psychoactive delta-9-tetrahydrocannabinol (THC) and non-psychoactive cannabidiol (CBD) [3]. The risk of developing manic symptoms in patients with bipolar disorder who use dronabinol, a synthetic version of THC administered orally, is largely unknown.

\section{Clinical Case}

Our patient is a 55-year-old Caucasian male who has been following with psychiatry since July of 2016 for substance use disorder and the following mental health conditions: bipolar I disorder, generalized anxiety disorder, PTSD, and intermittent sleep disturbances with no history of sleep apnea but recent reports of restless legs syndrome. Other relevant medical conditions include HIV and a history of primary restrictive eating disorder. He has no history of inpatient psychiatric hospitalizations or of suicide attempts. In terms of substance use he has a history of alcohol, cocaine, and cannabis use disorder but has been abstinent from alcohol, tobacco, and illicit substances for more than a year. The patient's relevant medication list includes: bupropion XL 150 $\mathrm{mg}$ daily, quetiapine $300 \mathrm{mg}$ daily at bedtime, and trazodone 50-100 mg at bedtime.

As a result of his HIV our patient was struggling with wasting syndrome and significant weight loss for which he was prescribed dronabinol $2.5 \mathrm{mg}$ twice daily on 5/19/17. At his visit with his psychiatrist on $7 / 10 / 17$, his bipolar disorder was noted to be stable. His dose of dronabinol was later increased on $7 / 21 / 17$ to $5 \mathrm{mg}$ twice daily. At his psychiatrist visit on $8 / 1 / 2017$, the patient was found to be in a state of hypomania, presenting with symptoms including: increased interest in sex, insomnia and increased animation. His judgment and impulse control were also noted to be slightly impaired at this visit. Excluding the dronabinol dose increase, no other medication changes had taken place and the patient was not using any alcohol or other substances.

To treat the patient's hypomania, quetiapine was discontinued and olanzapine $10 \mathrm{mg}$ at bedtime was started to also help with insomnia and promote weight gain. Bupropion was discontinued, his trazodone was tapered off, and dronabinol was also discontinued under suspicion for causing the hypomania. Upon follow up within a month, our patient's hypomania symptoms had mostly resolved. He had also begun gaining weight with the olanzapine and reported improved sleep. $\mathrm{He}$ acknowledged having a feeling of "buzz" while he had been taking the dronabinol. He was continued on olanzapine $10 \mathrm{mg}$ at bedtime and continued off the trazodone, bupropion and dronabinol. He continues to remain abstinent from alcohol and illicit drugs.

\section{Discussion}

The underlying mechanism of dronabinol induced manic symptoms in those with bipolar disorder remains unclear but may involve dopamine. Sensitization of the dopaminergic system by THC is thought to be associated with the development of manic symptoms in those that use cannabis [2]. THC is associated with increased dopaminergic cell firing, dopamine synthesis, and dopamine release when used acutely [4].

Other medications have been associated with causing manic symptoms, including bupropion and trazodone, as relevant to our case $[5,6]$. However, our patient had previously been stable on these medications prior to the addition of dronabinol. Thus, it is reasonable to conclude that the dronabinol likely caused our patient's hypomania symptoms.

${ }^{\star}$ Correspondence to: Audrey Umbreit, PharmD, Mayo Clinic Health System Eastridge Clinic, 101 Martin Luther King Jr Drive, Mankato, MN, 56001, USA, E-mail: Umbreit.Audrey@mayo.edu

Received: January 16, 2019; Accepted: January 30, 2019; Published: February 04, 2019 
In recent years the regulatory status of marijuana for medical and recreational use has changed in several states [7]. This has led to a growing population of patients who are using cannabinoids and as our case report has shown, those with underlying mental health conditions may be at risk of developing serious adverse effects. A thorough evaluation of mental health conditions and substance use disorders is necessary to identify patients for whom the use of medical cannabis or dronabinol is appropriate.

\section{Disclosures}

Dr. Sinha has received grant funding from UCare. Drs. Umbreit and Sieberg have no disclosures.

\section{Funding}

No funding received for this case report.

\section{References}

1. Whiting PF, Wolff RF, Deshpande S, Di Nisio M, Duffy S, et al. (2015) Cannabinoids for Medical Use: A Systematic Review and Meta-analysis. JAMA 313: 2456-2473. [Crossref]

2. Gibbs M, Winsper C, Marwaha S, Gilbert E, Broome M, et al. (2015) Cannabis use and mania symptoms: a systematic review and meta-analysis. $J$ Affect Disord 171: 39-47. [Crossref]

3. Lim K, See YM, Lee J (2017) A Systematic Review of the Effectiveness of Medical Cannabis for Psychiatric, Movement and Neurodegenerative Disorders. Clin Psychopharmacol Neurosci 15: 301-312. [Crossref]

4. Bloomfield MA, Ashok AH, Volkow ND, Howes OD (2016) The effects of I"9tetrahydrocannabinol on the dopamine system. Nature 539: 369-377. [Crossref]

5. Goren JL, Levin GM (2000) Mania with bupropion: a dose-related phenomenon? Ann Pharmacother 34: 619-621. [Crossref]

6. Warren M, Bick PA (1984) Two case reports of trazodone-induced mania. Am J Psychiatry 141: 1103-1104. [Crossref]

7. http://www.ncsl.org/research/health/state-medical-marijuana-laws.aspx

Copyright: (2019 Sinha S. This is an open-access article distributed under the terms of the Creative Commons Attribution License, which permits unrestricted use, distribution, and reproduction in any medium, provided the original author and source are credited. 